\title{
Editorial: Game Theory for 5G Wireless Networks
}

\author{
Haijun Zhang ${ }^{1}$ - Chunxiao Jiang ${ }^{2}$ - Julian Cheng ${ }^{3} \cdot$ Mugen Peng $^{4} \cdot$ Victor C. M. Leung ${ }^{5}$
}

Published online: 24 May 2017

(C) Springer Science+Business Media New York 2017

\section{Editorial}

Driven by the rapid development of wireless terminal equipments and wide usage of bandwidth-hungry mobile Internet applications, wireless data traffic is increasing in an exponential manner. which intend to integrate and benefit from many recent technical advances including ultra dense network (UDN), cloud radio access network (C-RAN), heterogeneous small cell networks, interference management and resource allocation, software defined wireless networks (SDN), selforganizing networks, cognitive radio, millimeter wave, massive multiple input multiple output (MIMO), and so on.

Haijun Zhang

haijunzhang@ieee.org

Chunxiao Jiang

jchx@tsinghua.edu.cn

Julian Cheng

julian.cheng@ubc.ca

Mugen Peng

pmg@bupt.edu.cn

Victor C. M. Leung

vleung@ece.ubc.ca

1 Beijing Engineering and Technology Research Center for Convergence Networks and Ubiquitous Services, University of Science and Technology Beijing, Beijing 100083, China

2 Tsinghua University, 30 Shuangqing Rd, Haidian Qu, Beijing Shi, China

3 School of Engineering, The University of British Columbia, Kelowna, BC V1V 1V7, Canada

4 Beijing University of Posts \& Telecommunications, 10 Xitucheng Rd, BeiTaiPingZhuang, Haidian Qu, Beijing Shi, China

5 Department of Electrical and Computer Engineering, The University of British Columbia, Vancouver, BC V6T 1Z4, Canada
Specifically, UDN can also offload the wireless data traffic of user equipments from macrocells, especially for an indoor environment where more than $80 \%$ of the data traffic occurs. C-RAN is also a promising wireless network architecture in $5 \mathrm{G}$ networks. In heterogeneous small cell networks, low power small cells (such as picocell and femtocell) when overlaid on top of the macrocells, can improve the coverage and capacity of cellular networks by exploiting spatial reuse of the spectrum. However, there are still many challenges and problems in $5 \mathrm{G}$ networks. Game theory is a powerful tool for modeling and analyzing the challenging problems in $5 \mathrm{G}$ wireless networks, which can be classified as cooperative games and non-cooperative games. Different game theories are suitable for various scenarios of $5 \mathrm{G}$ wireless networks in interference mitigation, resource allocation, spectrum access, economic analysis, etc.

This special issue features six selected papers with high quality. The first article titled "Tracking areas planning based on community detection in heterogeneous and small cell networks" proposes a tracking area (TA) planning method based on cooperative game for heterogeneous cellular networks in order to reduce the signaling overhead, improve the performance of calling successes and system utilizations while maintaining the offloading abilities of small cells.

The second article titled "Joint Resource Block and Power Allocation for Interference Management in Device to Device Underlay Cellular Networks: A Game Theoretic Approach" proposes a two-stage game theoretic approach for the problem of joint resource block (RB) and uplink transmission power allocation in a D2D underlay cellular network and confirms that the framework achieves to mitigate the interference of every link in the network while every link satisfies its SINR requirement.

In the next article with the title "Dormancy Mechanism Based Power Allocation in Heterogeneous Networks: A Stackelberg Game Approach", the authors studied dormancy 
mechanism of smallcells to improve energy efficiency and mitigate interference in two-tier heterogeneous networks (HetNets). By considering both the cross-tier interference and the smallcell dormancy mechanism, a power optimization oriented Stackelberg game considering dormancy mechanism for the downlink of two-tier HetNets were proposed to improve the smallcell network throughput and meanwhile dramatically reduce smallcells transmit power while increase smallcells performance in dense smallcell networks.

Wireless network virtualization (WNV) is widely regarded as one of the most promising technologies for $5 \mathrm{G}$ wireless communications. The fourth article titled "Equilibrium Price and Dynamic Virtual Resource Allocation for Wireless Network Virtualization" proposed an effective virtual resource (VR) allocation scheme for WNV from the perspective of the market-equilibrium theory. Considering the infrastructure providers (InPs) and the virtual network operators (VNOs) are willing to maximize its own benefit, the VR allocation problem is formulated as a multi-objective optimization problem. The effectiveness of the proposed VR allocation scheme has also been testified through extensive experiments.

Device-to-device (D2D) communication as an underlay coexistence with cellular networks allows mobile devices in close proximity to communicate directly, which offloads the cellular traffic. Relaying with D2D communications can further enhance the system performance and may promote the development of $5 \mathrm{G}$. The fifth article, which is entitled "Moderate Incentive Design for Delay-Constrained Deviceto-Device Relaying", proposed a moderate incentivecompatible data forwarding mechanism based on a Markov decision process framework with the principal-agent model. With implementation consideration, the authors proposed a greedy algorithm which only requires the past information. Compared with the existing inventive mechanisms, the proposed algorithm achieves a better performance on system utility.

C-RAN is recognized as one of the key enabling techniques for $5 \mathrm{G}$ due to its advantages in flexibility. In addition, the greatly increased energy efficiency (EE), which is evaluated by bits/Hz/J, is listed as one main objective when designing 5G wireless network. The last article titled "Mobile Networks and Applications Energy Efficient Clustering and Beamforming for Cloud Radio Access Networks" concentrated on EE optimization through C-RAN enabled flexible multicell cooperative transmission. The authors proposed a hierarchical iterative framework to solve the problem. Coalition formation game theory and fractional programming are utilized to obtain the optimal network cluster partition and beamformers respectively. Simulation results demonstrate the superior performance of the proposed algorithms.
Acknowledgements The guest editors are thankful to our reviewers for their effort in reviewing the manuscripts. We also thank the Edit-in-Chief, Dr. Imrich Chlamtac for his supportive guidance during the entire process. The special issue is sponsored by NSFC (Grant No. 61471025), the Chinese Association of Science and Technology Young Elite Scientist Sponsorship Program, and the Chinese Fundamental Research Funds for the Central Universities.

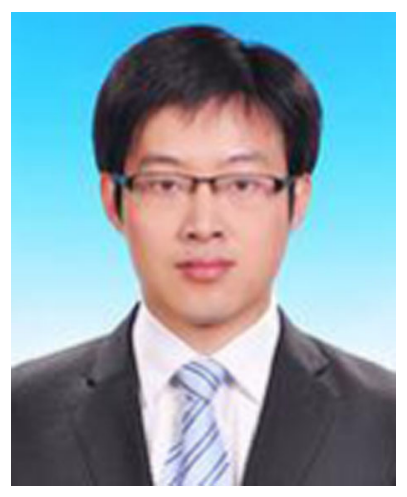

Haijun Zhang is currently a Full Professor in University of Science and Technology Beijing, China. He was a Postdoctoral Research Fellow in Department of Electrical and Computer Engineering, the University of British Columbia (UBC), Vancouver Campus, Canada. He received his Ph.D. degree in Beijing University of Posts Telecommunications (BUPT). From 2011 to 2012, he visited Centre for Telecommunications Research, King's College London, London, UK, as a Visiting Research Associate. Dr. Zhang has published more than 80 papers and authored 2 books. He serves as Editor of IEEE 5G Tech Focus, Journal of Network and Computer Applications, Wireless Networks, Telecommunication Systems, and KSII Transactions on Internet and Information Systems, and serves/served as leading Guest Editor for IEEE Communications Magazine, IEEE Transactions on Emerging Topics in Computing and ACM/Springer Mobile Networks \& Applications. He serves/served as General Co-Chair of 5GWN'17 and GameNets'16, Track Chair of ScalCom2015, Symposium Chair of the GameNets'14, and CoChair of Workshop on 5G Ultra Dense Networks in ICC 2017. He has served as a TPC member in a numerous international conferences. Prof. Zhang received the IEEE ComSoc Young Author Best Paper Award in 2017. His current research interests include 5G, Ultra-Dense Networks, Small Cells, LTE-U and Network Slicing. He is a senior member of IEEE.

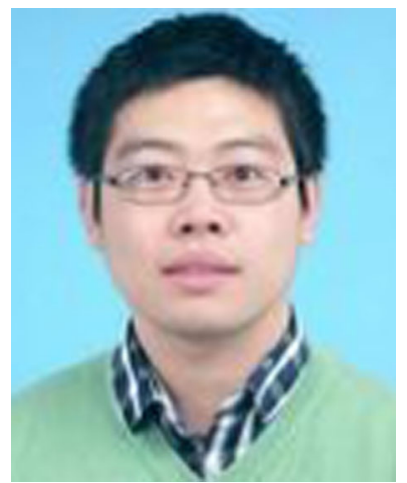

Chunxiao Jiang received the B.S. in information engineering from Beihang University in Jun. 2008 and the Ph.D. in electronic engineering from Tsinghua University in Jan. 2013, both with the highest honors. From Feb. 2013 - Jun. 2016, Dr. Jiang was a Postdoc in the Department of Electronic Engineering Tsinghua University, during which he visited University of Maryland College Park and University of Southampton. Currently, he is an assistant research fellow in Tsinghua Space Center, Tsinghua University, Beijing, China. Dr. Jiang has authored/co-authored over 70 referred IEEE journal papers. He is a recipient of the IEEE Globecom Best Paper Award in 2013, the IEEE GlobalSIP Best Student Paper Award in 2015, and the IEEE Communications Society Young Author Best Paper Award in 2017. Since 2015, Dr. Jiang became a IEEE Senior Member. His research interests lie in resource optimizations in space networks, terrestrial-satellite communication networks, social Networks, and big data privacy. 


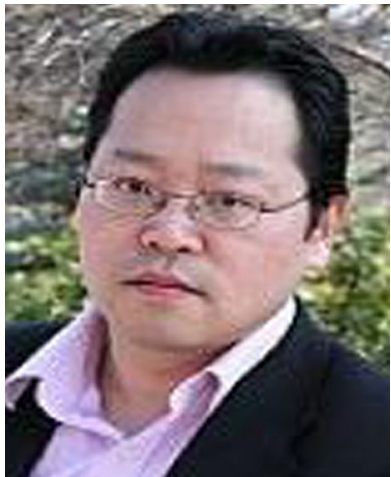

Julian Cheng received a B. Eng. Degree (First Class) in electrical engineering from the University of Victoria, Victoria, BC, Canada in 1995, a M.Sc. (Eng.) degree in mathematics and engineering from Queen's University, Kingston, ON, Canada in 1997, and a $\mathrm{PhD}$ degree in electrical engineering from the University of Alberta, Edmonton, AB, Canada, in 2003. Dr. Cheng was the recipient of numerous scholarships during his undergraduate and graduate studies, which included a President Scholarship from the University of Victoria and a postgraduate scholarship from the Natural Sciences and Engineering Research Council of Canada (NSERC). He was also a winner of the 2002 NSERC Postdoctoral Fellowship competition. Dr. Cheng held an Adjunct Professor position with the University of Alberta (2005-2010). $\mathrm{He}$ is a registered Professional Engineer in British Columbia. Dr. Cheng was also the founding chair of IEEE Okanagan Subsection. He is serving as Vice President and Treasurer for the Canadian Society of Information Theory (CSIT).

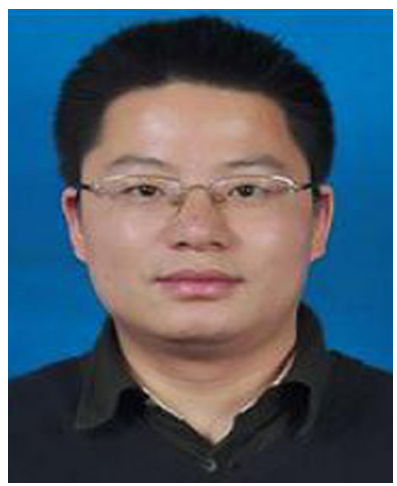

Mugen Peng received the B.E. degree in electronics engineering from the Nanjing University of Posts and Telecommunications, Nanjing, China, in 2000, and the $\mathrm{Ph} . \mathrm{D}$. degree in communication and information systems from the Beijing University of Posts and Telecommunications (BUPT), Beijing, China, in 2005. Afterward, he joined BUPT, where he has been a Full Professor with the School of Information and Communication Engineering since 2012. In 2014, he was an Academic Visiting Fellow with Princeton University, Princeton, NJ, USA. He leads a Research Group focusing on wireless transmission and networking technologies with the Key Laboratory of Universal Wireless Communications (Ministry of Education), BUPT. His main research areas include wireless communication theory, radio signal processing, and convex optimizations, with a particular interests in cooperative communication, self-organization networking, heterogeneous networking, cloud communication, and internet of things. He has authored/coauthored over 60 refereed IEEE journal papers and over 200 conference proceeding papers. Dr. Peng was a recipient of the 2014 I.E. ComSoc AP Outstanding Young Researcher Award, and the best paper award in IEEE WCNC 2015, WASA 2015, GameNets 2014, IEEE CIT 2014, ICCTA 2011, IC-BNMT 2010, and IET CCWMC 2009. He received the First Grade Award of the Technological Invention Award in the Ministry of Education of China, and the First Grade Award of Technological Invention Award from the China Institute of Communications. He is on the Editorial/Associate Editorial Board of the IEEE Communications Magazine, IEEE Access, IEEE Internet of Things Journal, IET Communications, and China Communications. He has been the guest leading editor for the special issues in the IEEE Wireless Communications. He is the Fellow of IET.

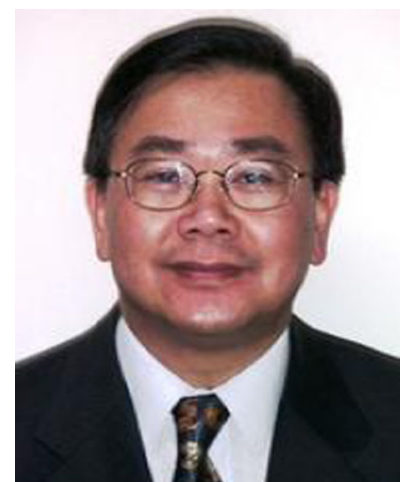

Victor C. M. Leung received the BASc (Hons.) in electrical engineering from the UBC in 1977 , and was awarded the APEBC Gold Medal as the head of the graduating class in the Faculty of Applied Science. He attended graduate school at $\mathrm{UBC}$ on a Natural Sciences and Engineering Research Council Postgraduate Scholarship and completed his $\mathrm{PhD}$ in electrical engineering in 1981. From 1981 to 1987 , he was a Senior Member of Technical Staff at MPR Teltech, Burnaby, Canada. In 1988, he was a Lecturer in Electronics in the Chinese University of Hong Kong. He returned to UBC as a faculty member in 1989 , where he is currently a Professor and the inaugural holder of the TELUS Mobility Research Chair in Advanced Telecommunications Engineering. His research interests are in the general areas of wireless networks and mobile systems, where he has co-authored more than 1000 conference/journal articles and book chapters. Dr. Leung is a registered member of the Association of Professional Engineers and Geoscientists of British Columbia. He is a Fellow of IEEE, a Fellow of the Engineering Institute of Canada, a Fellow of the Canadian Academy of Engineering, and a Fellow of the Royal Society of Canada. He was a Distinguished Lecturer of the IEEE Communications Society. He has served on the editorial boards of the IEEE Journal on Selected Areas in Communications, IEEE Transactions on Wireless Communications, IEEE Transactions on Computers, IEEE Transactions on Vehicular Technology, and is serving on the editorial boards of IEEE Wireless Communications Letter, IEEE Transactions on Green Communications and Networking, IEEE Access, and several other journals. He has provided leadership to the Technical Program Committees and Organization Committees of many conferences. Dr. Leung received the IEEE Vancouver Section Centennial Award and 2012 UBC Killam Research Prize. He is the recipient of the 2017 Canadian Award for Telecommunications Research. He co-authors a paper in the Feb. 2014 issue of IEEE Communications Magazine, which has been selected for the 2017 I.E. ComSoc Fred W. Ellersick Prize. 